\title{
Transvesical Endoscopic Peritoneoscopy: Intra-abdominal Scarless Surgery for Urologic Applications
}

\author{
Estevao Lima, MD, FEBU, Carla Rolanda, MD, \\ and Jorge Correia-Pinto, $M D, P h D$
}

\author{
Corresponding author \\ Jorge Correia-Pinto, MD, PhD \\ Instituto de Ciências da Vida e Saúde (ICVS), \\ Escola de Ciências da Saúde, Universidade do Minho, \\ Campus de Gualtar; 4709-057 Braga, Portugal. \\ E-mail: jcp@ecsaude.uminho.pt \\ Current Urology Reports 2008, 9:50-54 \\ Current Medicine Group LLC ISSN 1527-2737 \\ Copyright $(0) 2008$ by Current Medicine Group LLC
}

For many abdominal procedures, advantages such as minimal scarring, reduced pain, and faster recovery have made laparoscopy the favored approach over traditional open surgery. The most recent minimally invasive approach is natural orifice transluminal endoscopic surgery (NOTES), which limits morbidity because this surgery does not require incision. This article reviews the history, development, and current and future applications of NOTES in the field of urology.

\section{Introduction}

The introduction of laparoscopic surgery at the end of the $20^{\text {th }}$ century revolutionized modern surgical practice, significantly changing surgical considerations, techniques, and all other aspects of modern surgical patient care [1]. Simultaneously, endoscopy evolved from simple diagnostic to progressively invasive therapeutic procedures [2]. Historically, endoscopy has avoided gastrointestinal lumen perforation; although this principle mostly persists, recent anecdotal reports have described safe endoscopic procedures beyond the visceral wall, such as transgastric drainage of pancreatic pseudocyst [3]. Reddy and Rao [4], for example, performed transgastric appendectomy in humans cases, while Kalloo et al. [5••] demonstrated the feasibility and surgical utility of transgastric access to the peritoneal cavity in a porcine model.

These developments marked a new era in minimally invasive abdominal surgery, compelling novel, previously audacious goals, such as avoidance of incisions and pain. With the visceral wall no longer a barrier to endoscopic intervention, various authors described novel, complex abdominal procedures [6-16]. In 2006, such reports motivated researchers in this emerging field to organize the Natural Orifice Surgery Consortium for Assessment and Research (NOSCAR), a joint initiative supported by the American Society for Gastrointestinal Endoscopy (AGES) and the Society of American Gastrointestinal and Endoscopic Surgeons (SAGES). Proceedings were reported in the Natural Orifice Transluminal Endoscopic Surgery (NOTES) white paper [17••]. European researchers also formed the European Association of Transluminal Surgery (http://www.eats.fr) and the EURO-NOTES Foundation (http://www.euro-notes.eu) to ease cooperation between the European Association for Endoscopic Surgery (EAES) and the European Society of Gastrointestinal Endoscopy (ESGE), which focuses on NOTES-related activities. As a result, an increasing number of researchers are committed to NOTES, publishing experimental reports and attending industry-supported, international conferences where specialists confirm the approach's feasibility and efficacy for abdominal surgery.

The use of natural orifices for abdominal surgery entrance ports subsequently lead to transvesical access, resulting in unexpected success for abdominal urologic and nonurologic procedures $[18 \bullet \bullet]$.

This article reviews the current status of NOTES, focusing on new challenges and potential clinical applications in urology.

\section{Current Status of NOTES}

\section{Rationale and potential benefits}

NOTES is a revolutionary peritoneal cavity intervention; a natural convergence of intraluminal (endoscopy) and extraluminal (laparoscopy) endoscopic techniques [19]; a third-generation surgery after open surgery (first generation) and laparoscopy (second generation) $[16,18 \bullet \bullet, 20]$ that requires new equipment, special training, and often interdisciplinary collaboration. Advantages over other approaches include no scarring, less anesthesia and postoperative pain, no abdominal wound infection or 
incisional hernia, fewer adhesions and postoperative ileus, and more rapid recovery $[17 \bullet \bullet]$. However, no studies have demonstrated advantages beyond cosmetic appearance. Studies need to compare the efficacy of NOTES and laparoscopy in terms of surgical stress prevention and morbidity. Impact on immune system also needs to be determined. Until these issues are addressed, potential benefits remain theoretical.

\section{Experimental studies}

Natural orifice surgery began in 1928 when Decker [21] performed the first culdoscopy. In 2002, Gettman et al. [22] reported a transvaginal laparoscopic nephrectomy in five female pigs using a single $5-\mathrm{mm}$ abdominal trocar; however, limitations related to the porcine model and instrumentation made the procedure cumbersome. Kalloo et al. $[5 \bullet \cdot]$ reported the first natural orifice endoscopic surgery using a transgastric approach in a porcine model in which they orally introduced a flexible endoscope into the peritoneal cavity to perform peritoneoscopy and liver biopsies. At procedure's end, researchers closed the gastric wall with endoscopic clips. In five experiments, all pigs recovered and gained weight.

Several studies have since used the transgastric port for intraperitoneal abdominal procedures, such as fallopian tube ligation, cholecystectomy, gastrojejunostomy, lymphadenectomy, oophorectomy, partial hysterectomy, splenectomy, appendectomy, and diaphragmatic pacing [6-15,23]. Following the initial enthusiasm, however, abdominal procedures through isolated transgastric routes raised limitations that jeopardized application in humans. Potential barriers to clinical practice included safe access to peritoneal cavity; gastric closure; infection prevention; spatial orientation; stable multitasking platform to obtain adequate anatomy exposure, organ retraction, secure grasping, and triangulation; difficulty in controlling the pneumoperitoneum; and management of iatrogenic intraperitoneal complications. These limitations are primarily related to the isolated transgastric approach and the characteristics of endoscopes such as flexibility $[17 \bullet \bullet]$.

Lima et al. [18 $\bullet$ ] hypothesized that a port enabling rigid instrument use would be advantageous, and subsequently assessed the feasibility and safety of a $5-\mathrm{mm}$ transvesical port for abdominal cavity access. This approach was feasible and safely performed in a survival porcine model $[18 \bullet \bullet]$. Recently, the same researchers used the transvesical port to perform thoracoscopy with lung biopsies through the diaphragm in a survival porcine model study [24]. A transcolonic port approach has also been reported involving several complications related to the nonsterile nature of the colon [25].

Cholecystectomy is the most challenging isolated transgastric approach. Using two endoscopes, or a single endoscope conjugated with a transabdominal trocar, Park et al. [7] and Swanstrom et al. [26] experienced significant difficulties performing cholecystectomy using shape-lock technology. Rolanda et al. [20] introduced a combined transgastric and transvesical approach to effectively perform moderately complex upper-abdominal procedures such as cholecystectomy. More recently, Lima et al. [16] used the same combined approach to perform nephrectomy. In fact, this was the first group to combine distinct natural orifices approaches to perform complex abdominal surgery.

\section{Human studies}

Reddy and Rao [4] proved the transgastric approach feasibility by performing a NOTES appendectomy in humans [4]. Recently, Marescaux et al. [27] performed a transvaginal cholecystectomy using a single abdominal 2-mm trocar. Although unpublished, Brazilian researchers claim to have performed cholecystectomy by a hybrid transvaginal and transabdominal trocar approach. More recently, the first human cases of transgastric cholecystectomy have been presented using a single transabdominal trocar. Further, initial clinical evaluation of the bladder as a NOTES port has already been successfully attempted in humans [28].

\section{Current limitations}

NOTES is still in an investigational, developmental stage. Several limitations need to be addressed before these procedures are safe and reliable. NOSCAR has established taxonomy, delineated the current limitations of NOTES, and instigated a unified plan of research to propel NOTES into human practice. Challenges include creating a safe, transmural access point; maintaining sterility of peritoneal cavity despite peroral instrument use; creating a pneumoperitoneum to distend the peritoneal cavity and allow adequate visualization; ensuring availability of endoscopes and equipment for therapeutic procedures; and developing an entirely reliable means of securely closing the transmural access point $[17 \bullet \bullet]$. Of these barriers to clinical practice, gastrostomy closure is the most crucial to solve; various devices are being developed to aid closure [29]. Fast, simple, and effective endoscopic suturing devices will be important for anastomosis, closure of access incisions, and treatment of complications such as bleeding. Examples of endoscopic suturing devices include the prototype known as the Eagle Claw (Olympus America, Center Valley, PA); the novel endoscopic incision and closure device called the Stringer Device (LSI Solutions, Victor, NY); the g-Prox (USGI Medical, San Clemente, CA), which combines an aggressive grasper with a needle delivery device that delivers expandable baskets connected by permanent suture; and a prototype device that combines the well-known technology of T-fasteners with a locking cinch device (Johnson and Johnson, New Brunswick, NJ) [29,30]. Currently, endoclips are the most commonly used method for gastrostomy closure $[5 \bullet \bullet$. However, endoclips are designed for hemostasis and are not sited for approximating gastrostomy edges for incision closure. 
Unless safe and simple devices with proven efficacy for endoscopic closure are available, NOTES will remain in the research field due to an unacceptable complication risk in general practice. Availability of a safe and simple gastrostomy closure device is therefore essential for widespread adoption.

\section{The Role of Urology in NOTES Bladder as natural orifice}

As with the transgastric port innovation, the transvesical approach surpassed a classical urologic barrier, the urinary tract wall. Bladder wall perforation was considered a potential complication of urologic procedures. However, with success similar to that of transgastric port, the bladder is an attractive port for NOTES procedures once sterilized because it allows passage of rigid instruments and it is the most anterior access (above bowel loops) from the lower abdomen $[18 \bullet \bullet]$.

\section{Technique}

Until recently, experimental and human preliminary studies reported that the transvesical port was easy to install, even with instruments for urologic purposes, using a technique based on the Seldinger principle. We use a ureteroscope introduced through the urethra into the bladder with pneumodistension, emptying urine from the bladder and distending it with $\mathrm{CO}_{2}$. The vesicotomy site is carefully selected on the bladder dome. A mucosal incision is made with scissors introduced through the working channel of the ureteroscope. Subsequently, a 5 Fr open-ended ureteral catheter is pushed through the incision into the peritoneal cavity. A 0.035 -inch flexible-tip guidewire is inserted into the peritoneal cavity through the ureteral catheter lumen. Guided by the flexible-tip guide-wire, a ureteroscope sheath dilator enlarges the vesical port with a flexible 5.5-mm overtube enveloping it. Introduction of a ureteroscope via overtube into the peritoneal cavity establishes a pressure-controlled $\mathrm{CO}_{2}$ pneumoperitoneum. The ureteroscope is ultimately withdrawn from the abdominal cavity after $\mathrm{CO}_{2}$ removal and a Foley catheter is inserted into the bladder for 3 to 4 days $[18 \bullet \bullet, 24]$.

\section{Advantages and limitations}

Lower abdominal access points, including the transvesical, transvaginal, and transcolonic ports, can be used as an isolated or complementary transgastric port. Several researchers who performed complex abdominal procedures via an isolated transgastric pathway have encountered problems with decreased triangulation and retroflexion [26]. Lower abdominal ports, however, may overcome some of these limitations, with added advantage in complex operations. Although lower abdominal ports provide frontal access to upper abdominal organs and enable improved instrument access, the transvesical port offers sterility and the anatomical advantage of the most anterior lower abdominal access. Further, the transvesical port enables rigid instrument introduction and does not necessarily require closure [31].

Accessing the peritoneal cavity through a natural orifice risks damaging adjacent organs during visceral wall incision. Among natural orifice ports, transvesical port creation with a Seldinger-like technique may be the safest approach because bowel loops that contact the bladder wall are unrestricted in the abdomen, which make them run away from bladder instruments. Further, in procedures involving two natural orifices, such as transgastric and transvesical, the transvesical image can easily monitor the transgastric creation.

The transvesical port procedure involves a significant challenge related to instrument size, limiting organ retrieval through this port. Another concern related to transvesical port use is the necessity of bladder closure. Vesical perforation involves potential complications, such as peritoneal urine leakage with secondary infection (peritonitis), that commonly occur as delayed complications of undiagnosed traumatic vesical perforation or pathological bladder conditions, such as neoplasms. Experiments in pigs demonstrated that $5 \mathrm{~mm}$ bladder hole closure is not absolutely necessary if bladder drainage is assured $[18 \bullet \bullet, 24]$. However, development of a closure device or method is imperative for the widespread adoption of transvesical port option in NOTES.

\section{Applications in urology}

NOTES' urologic application may not be immediately apparent, considering the current acceptance of laparoscopic surgery. However, several groups are using natural orifices to perform complex procedures such as nephrectomy.

Gettman et al. [22] first performed a complete transvaginal laparoscopic dissection and nephrectomy in a porcine model using a single, 5 - $\mathrm{mm}$ abdominal trocar for visualization. A completely transvaginal laparoscopic nephrectomy was performed once, but limitations imposed by porcine anatomy and available instrumentation hindered the procedure [22]. More recently, Clayman et al. [32] performed a porcine nephrectomy with a single $12-\mathrm{mm}$ trocar placed in the midline and the transvaginal introduction of a TransPort Multi-Lumen Operating Platform (USGI Medical, San Clemente, CA). This flexible device has four working channels and can be locked into position, creating a rigid multitasking platform that enables two-handed tissue manipulation [32]. Further, Lima et al. [16] demonstrated the feasibility of NOTES nephrectomy. In a nonsurvival study, combined transgastric and transvesical approaches were established in six female pigs. Under ureteroscope visualization through a $5-\mathrm{mm}$ transvesical port, researchers controlled the orally introduced flexible gastroscope by the gastrotomy into the peritoneal cavity. Right or left nephrectomy were performed using instruments introduced by devices that worked in the renal hilum, alternating intervention on dissection or retraction procedures. In all 
animals, both kidneys were visualized, and the renal vessels and ureter were reasonably individualized and ligated separately with ultrasonic scissors introduced through the transvesical port [16].

Nephrectomy most likely will be one of the final renal procedures potentially performed by NOTES in the near future. However, much research is needed to minimize unexpected complications before NOTES can be applied in humans. Robotics and magnetically anchored instrumentation used to perform single trocar laparoscopic nephrectomy may be logical steps in the development of scarless abdominal surgery by NOTES [33].

Beyond renal procedures, other potential human applications of NOTES in urology include varicocelectomy, investigation of nonpalpable testis, and treatment of urachus remnants using transgastric or transvesical ports.

Involvement of urologists in other abdominal procedures NOTES may present a tremendous challenge for urologists in terms of technical demands. It may also involve multidisciplinary teams to deal with nonurologic clinical situations, considering the simplicity of accessing and viewing the upper abdominal organs via the transvesical port $[15,24]$.

In animal and human settings, the transvesical port enables feasible and useful peritoneoscopy of all intraabdominal viscera, mainly the upper abdominal organs $[18 \bullet \bullet, 24,28]$. Further, the transvesical port is gaining a place in NOTES as a unique port associated with the transgastric port [16,20]. Rolanda et al. [20] demonstrated the utility of a combined transgastric and transvesical approach, performing a reliable, feasible, exclusive NOTES cholecystectomy. This study emphasized the transvesical port's ability to overcome limitations reported in a cholecystectomy performed exclusively through a transgastric port [20].

Although possibly difficult to accept clinically, the transvesical port provides exceptional access to the upper abdominal organs and may enable a transdiaphragmatic endoscopic approach to the thoracic cavity in a long-term survival study in a porcine model [21].

Most of these studies are preliminary and only represent the birth of NOTES in urology; however, they demonstrate a need for new instruments and further research that provides evidence that experimental successes may advantageously translate to clinical practice in humans.

\section{Conclusions}

In many areas of medicine, divisions between specialties are blurring. For example, stent placement for carotid stenosis is now performed by neuroradiologists, interventional radiologists, vascular surgeons, and cardiologists. Similar reports commonly occur in other clinical areas. Currently, NOTES procedures and research are primarily performed by gastroenterologists and surgeons. However, because the mouth and colon are not the only access points, urologists and gynecologists have approached the peritoneum through the bladder and vagina. Further, NOTES has enabled transvaginal nephrectomy, transgastric adrenalectomy (unpublished data), peritoneoscopy, and thoracoscopy using a transvesical approach, and cholecystectomy and nephrectomy using a combined transvesical and transgastric approach $[16,18 \bullet \bullet, 20,22,24$, $28,32]$. These procedures have mostly been performed in animal models, but human application is pending.

Urologists have been encouraged in this developing field to meet an especially great demand should NOTES develop as some investigators have proposed. Further, the future may require the development of new NOTES training programs to address specific demands.

\section{Disclosures}

No potential conflict of interest relevant to this article was reported.

\section{References and Recommended Reading}

Papers of particular interest, published recently, have been highlighted as:

- Of importance

- Of major importance

1. Harrell AG, Heniford BT: Minimally invasive abdominal surgery: lux et veritas past, present, and future. Am J Surg 2005, 190:239-243.

2. Liu R, Chand B, Ponsky J: The future of surgical endoscopy. Endoscopy 2005, 37:38-41.

3. Vitale GC, Lawhon JC, Larson GM, et al.: Endoscopic drainage of the pancreatic pseudocyst. Surgery 1999, 26:616-623.

4. Richards WO, Rattner DW: Endoluminal and transluminal surgery: no longer if, but when. Surg Endosc 2005, 19:461-463.

5.• Kalloo AN, Singh VK, Jagannath SB, et al.: Flexible transgastric peritoneoscopy: a novel approach to diagnostic and therapeutic interventions in the peritoneal cavity. Gastrointest Endosc 2004, 60:114-117.

The first description of transgastric surgery. This manuscript marks the birth of NOTES.

6. Jagannath SB, Kantsevoy SV, Vaughn CA, et al.: Per-oral transgastric ligation of fallopian tubes with long-term survival in a porcine model. Gastrointest Endosc 2005, 61:449-453.

7. Park PO, Bergström M, Ikeda K, et al.: Experimental studies of transgastric gallbladder surgery: cholecystectomy and cholecystogastric anastomosis (videos). Gastrointest Endosc 2005, 61:601-606.

8. Kantsevoy SV, Jagannath SB, Niiyama H, et al.: Endoscopic gastrojejunostomy with survival in a porcine model. Gastrointest Endosc 2005, 62:287-292.

9. Bergström M, Ikeda K, Swain P, Park PO: Transgastric anastomosis by using flexible endoscopy in a porcine model. Gastrointest Endosc 2006, 63:307-312.

10. Fritscher-Ravens A, Mosse CA, Ikeda K, Swain P: Endoscopic transgastric lymphadenectomy by using EUS for selection and guidance. Gastrointest Endosc 2006, 63:302-306

11. Wagh MS, Merrifield BF, Thompson CC: Survival studies after endoscopic transgastric oophorectomy and tubectomy in a porcine model. Gastrointest Endosc 2006, 63:473-478. 
12. Merrifield BF, Wagh MS, Thompson CC: Peroral transgastric organ resection: a feasibility study in pigs. Gastrointest Endosc 2006, 63:693-697.

13. Kantsevoy SV, Hu B, Jagannath SB, et al.: Transgastric endoscopic splenectomy: is it possible? Surg Endosc 2006, 20:522-525.

14. Sumiyama K, Gostout CJ, Rajan E, et al.: Pilot study of the porcine uterine horn as an in vivo appendicitis model for development of endoscopic transgastric appendectomy. Gastrointest Endosc 2006, 64:808-812.

15. Onders R, McGee MF, Marks J, et al.: Diaphragm pacing with natural orifice transluminal endoscopic surgery: potential for difficult-to-wean intensive care unit patients. Surg Endosc 2007, 21:475-479.

16. Lima E, Rolanda C, Pêgo JM, et al.: Third generation nephrectomy by natural orifices translumenal endoscopic surgery. J Urol 2007, In press.

17.• Rattner D, Kalloo A, and SAGES/ASGE working group on NOTES. ASGE/SAGES working group on natural orifice translumenal endoscopic surgery. Surg Endosc 2006, 20:329-333.

This interdisciplinary manuscript established the fundamental challenges to the safe introduction of NOTES.

18.• Lima E, Rolanda C, Pêgo JM, et al.: Transvesical endoscopic peritoneoscopy: A novel $5 \mathrm{~mm}$ port for intraabdominal scarless surgery. J Urol 2006, 176:802-805.

Includes the first description of the transvesical port approach and represents the beginning of the urology commitment to NOTES.

19. Malik A, Mellinger JD, Hazey JW, et al.: Endoluminal and transluminal surgery: Current status and future possibilities. Surg Endosc 2006, 20:1179-1192.

20. Rolanda C, Lima E, Pêgo JM, et al.: Third-generation cholecystectomy by natural orifices: transgastric and transvesical combined approach (with video). Gastrointest Endosc 2007, 65:111-117.

21. Decker A: Culdoscopy: a method for visual diagnosis of gynecologic disease. Clin Symp 1952, 4:201-210.

22. Gettman MT, Lotan Y, Napper CA, Cadeddu JA: Transvaginal laparoscopic nephrectomy: development and feasibility in the porcine model. Urology 2002, 59:446-450.
23. Sumiyama K, Gostout CJ, Rajan E, et al.: Transgastric cholecystectomy: transgastric accessibility to the gallbladder improved with the SEMF method and a novel multibending therapeutic endoscope. Gastrointes Endosc 2007, 65:1028-1034.

24. Lima E, Henriques-Coelho T, Rolanda C, et al.: Transvesical thoracoscopy: a natural orifice translumenal endoscopic approach for thoracic surgery. Surg Endosc 2007, 21:854-858.

25. Fong DG, Pai RD, Thompson CC: Transcolonic endoscopic abdominal exploration: a NOTES survival study in a porcine model. Gastrointest Endosc 2007, 65:312-318.

26. Swanstrom LL, Kozarek R, Pasricha PJ, et al.: Development of a new access device for transgastric surgery. J Gastrointest Surg 2005, 9:1129-1137.

27. Marescaux J. Operation Anubis: A new step in NOTES history! Available at http://www.websurg.com/notes. Accessed on September 13, 2007.

28. Gettman MT, Blute ML: Transvesical peritoneoscopy: initial clinical evaluation of the bladder as a portal for natural orifice translumenal endoscopic surgery. Mayo Clin Proc 2007, 82:843-845.

29. Sclabas GM, Swain P, Swanstrom LL: Endoluminal methods for gastrotomy closure in natural orifice transenteric surgery (NOTES). Surg Innov 2006, 13:23-30.

30. Ryou M, Pai RD, Sauer JS, et al.: Evaluating an optimal gastric closure method for transgastric surgery. Surg Endosc 2007, 21:677-680.

31. Rolanda C, Lima E, Correia-Pinto J: Searching the best approach for third-generation cholecystectomy. Gastrointest Endosc 2007, 65:354-355.

32. Clayman RV, Box GN, Abraham JB, et al.: Rapid communication: transvaginal single-port NOTES nephrectomy: initial laboratory experience. J Endourol 2007, 21:640-644.

33. Zeltser IS, Bergs R, Fernandez R, et al.: Single trocar laparoscopic nephrectomy using magnetic anchoring and guidance system in the porcine model. J Urol 2007, 178:288-291. 